\title{
Systematic risk in the biopharmaceutical sector: a multiscale approach
}

\author{
Gazi Salah Uddin ${ }^{1} \cdot$ Muhammad Yahya $^{2}$ (D) Stelios Bekiros ${ }^{3,4}$. \\ Raanadeva Jayasekera ${ }^{5} \cdot$ Gerhard Kling $^{6}$
}

Accepted: 29 October 2021

(c) The Author(s) 2021

\begin{abstract}
It is well documented that the biopharmaceutical sector has exhibited weak financial returns, contributing to underinvestment. Innovations in the industry carry high risks; however, an analysis of systematic risk and return compared to other asset classes is missing. This paper investigates the time-frequency interconnectedness between stocks in the biotech sector and ten asset classes using daily cross-country data from 1995 to 2019. We capture investors' heterogeneous investment horizons by decomposing time series according to frequencies. Using a maximal overlap discrete wavelet transform (MODWT) and a dynamic conditional correlation (DCC)-Student-t copula, diversification potentials are revealed, helping investors to reap the benefits of investing in biotech. Our findings indicate that the underlying assets exhibit nonlinear asymmetric behavior that strengthens during periods of turmoil.
\end{abstract}

Keywords OR in medicine $\cdot$ Biotech $\cdot$ Time-varying copulas $\cdot$ Wavelets $\cdot$ Risk management

$\bowtie$ Muhammad Yahya

muhammad.yahya@inn.no

Gazi Salah Uddin

gazi.salah.uddin@liu.se

Stelios Bekiros

Stelios.bekiros@um.edu.mt; stelios.bekiros@eui.eu

Raanadeva Jayasekera

jayasekr@tcd.ie

Gerhard Kling

gerhard.kling@abdn.ac.uk

1 Department of Management and Engineering, Linköping University, 58183 Linköping, Sweden

2 Department of Business Administration, Inland Norway University of Applied Sciences, 2418 Elverum, Lillehammer, Norway

3 Department of Banking and Finance, FEMA and DLT Centre, University of Malta, Msida, Malta

4 Department of Economics, European University Institute, Via delle Fontanelle, 18, I-50014 Florence, Italy

5 Trinity Business School, Dublin 2, Dublin, Ireland

6 Business School, University of Aberdeen, Edward Wright Building S29, Aberdeen, UK 
JEL Classification $\mathrm{C} 32 \cdot \mathrm{C} 44 \cdot \mathrm{G} 1 \cdot \mathrm{G} 10 \cdot \mathrm{G} 15 \cdot \mathrm{L} 65$

\section{Introduction}

The literature has documented mediocre financial returns in the biopharmaceutical sector accompanied by high risks due to drug pipelines and economic conditions (Fagnan et al., 2013; Fernandez et al., 2012; Gopalakrishnan et al., 2008). However, systematic risk and return compared to other asset classes have not been analyzed, which this paper addresses. Since Markowitz (1952) laid the foundations for the capital asset pricing model (CAPM) (Fabozzi \& Francis, 1978; Lintner, 1965; Mossin, 1966; Sharpe, 1964), systematic risk, measured by beta coefficients, has been estimated from the relationship between stock and market returns. A literature has emerged to derive time-varying betas using bivariate t-GARCH, Markov switching model, and Kalman filters (for an overview, see Mergner \& Bulla, 2008). Limitations such as focusing on a single factor driving risk have been addressed by multifactor models (Fama \& French, 1993). Other sources of risk stem from shocks in commodity prices, e.g., oil prices (Boyer \& Filion, 2007). In addition, gold serves as an anti-cyclical asset (Baur \& Lucey, 2010). This study includes oil and gold prices, focusing on their interconnectedness with the biopharmaceutical sector and other asset classes.

Investment horizons differ; hence, we apply a maximal overlap discrete wavelet transform (MODWT) to decompose short and long-term price movements. This approach is in line with recent studies by Aguiar-Conraria and Soares (2014), Kahraman and Unal (2016), and Mestre (2021). Mestre (2021) estimates a time-frequency multi-betas model using an AR-EGARCH with and without wavelets. Aguiar-Conraria and Soares (2014) illustrate wavelet coherency and phase differences between stock market returns and oil prices. In contrast, Kahraman and Unal (2016) apply Vector Autoregressive Moving Average models to predict metal prices. Our approach differs in terms of methodology as we apply dynamic conditional correlation (DCC)-Student-t copulas. In Operations Research, a large body of literature has applied MODWT to decompose time series. These studies conduct forecasting of financial time series (Jana et al., 2021), multiresolution analysis (Kilic and Ugur, 2018), risk assessments for different trading horizons (Tzagkarakis \& Maurer, 2020), and multifractal theory (Zhao et al., 2015) among many other applications. In addition, copulas have been used to study time-varying asymmetric tail dependence in portfolio selection (Yan et al., 2020) and the risk exposure to oil price shocks (Shahzad et al., 2021).

Our contribution is twofold. To the best of our knowledge, this is the first study to evaluate the connectedness of biotech assets with other asset classes. Specifically, we contribute by examining the potential of various biotech assets (Nasdaq biotech, DJGL US biotech, SP500 pharmaceuticals, and NYSE ARCA Tech) as safe havens in periods of turmoil of major stock market indices (S\&P500 composite, FTSE100, and STOXX Europe), commodities (crude oil and gold), and the forex market (Euro to USD exchange rate). Our methodological contribution refers to evaluating the temporal and spectral interdependence among asset classes employing a wavelet-based DCC-Student-t copula. We capture heterogeneous investment preferences of market participants by decomposing time series into frequency horizons. Applying a wavelet decomposition via an entropy analysis captures heterogeneous investment preferences, which are not apparent in the scale-dependent information set. In the context of wavelet analysis, the Shannon entropy might understate the randomness of the data. Consequently, the Wavelet Entropy is commonly used based on the energy distribution of wavelet coefficients. We refer to Zou et al. (2015) for further details. 
Market participants have heterogeneous investment and risk preferences, resulting in specific term objectives and investment horizons. The wavelet transforms analysis decomposes the time series into signals providing information embedded in the frequency domain. These signals are attributed to short-, medium-, and long-run components based on their frequencies. We utilize a time-varying Student-t copula framework to examine the temporal connectedness among asset classes. Copulas are flexible and efficient in modeling both average and extreme joint movements (tail dependence). Hence, the combination of wavelet decomposition and time-varying Student-t copula provides information that enhances our understanding of dependence among asset classes in periods of turmoil or stability. These findings are essential for risk assessment and portfolio management decisions over different investment horizons.

We use daily data sourced from DataStream from 1995 to 2019. This period includes several shocks, including the Asian Financial Crisis (1997-1998), the Iraq war (2003), the Global Financial Crisis (GFC) (2008-2009), the debt crisis in Europe (ESDC) (2010-2012), and the drop in crude oil prices in 2014. Copula estimates show that the dependence parameter is high between Nasdaq biotech and other biotech assets for both original and decomposed (i.e., split between short-medium- and long-term horizon) return series. Furthermore, the connectedness structure for Nasdaq biotech and financial indices also exhibits a moderate to strong dependence across all series. In contrast, linkages between Nasdaq biotech, commodities, and exchange rates are characterized by weak and negative dependence, indicating a strong potential to attain diversification benefits over various frequency horizons.

Furthermore, we examine investors' risk exposures deriving the value-at-risk (VaR), CoVar and $\triangle \mathrm{CoVaR}$ parameters (Adrian \& Brunnermeier, 2016). We find that over the pre-2000 period, the VaRs are higher for biotech assets, whereas, over the post-2000 period, the VaRs have significantly declined with few periods of abrupt changes, especially during the Iraq war and the GFC, in line with the findings of Thakor et al. (2017). Furthermore, we find that the VaRs have declined significantly with longer investment horizons, supporting the mean-reverting behavior of asset prices.

The remainder of the paper is structured as follows. Section 2 discusses the methodology followed by data and preliminary analyses in Sect. 3. Section 4 presents our empirical findings, followed by portfolio analyses in Sect. 5 . Section 6 concludes.

\section{Methodology}

\subsection{Maximal overlap discrete wavelet transforms}

Wavelet transform analysis decomposes a series into a set of components, which correspond to various frequency horizons. Hence, short-, medium-, and long-run movements can be established. We utilize a maximal overlap discrete wavelet transform (MODWT), which refers to a modified version of a discrete wavelet transform (DWT). MODWT does not suffer from shortcomings such as shift-invariance and dyadic length. ${ }^{1}$ MODWT is known by various names in the engineering and statistical literature, such as 'stationary DWT' (Nason \& Silverman, 1995), 'translation-invariant DWT' (Coifman \& Donoho, 1995), and 'time-invariant DWT' (Pesquet et al., 1994). This subsection provides a brief introduction to MODWT, and the interested readers are referred to Nason and Sachs (1999), Percival and Walden (2000), and Gençay et al. (2001) for further details.

$\overline{1}$ The discussion of drawbacks and advantage of DWT and MODWT is beyond the scope of this paper. 
As we work with financial time series, first-differencing, i.e., return series, results in stationary series, which we denote as $r_{t}$ with $t=1,2, \ldots, N$. We can deconstruct $r_{t}$ into a set of $J$ frequencies by circularly filtering $r_{t}$ by employing the MODWT wavelet and scaling filters (1), where, $\tilde{h}_{j, l}$ and $\tilde{g}_{j, l}$ represent the wavelet and scaling filters, respectively, and $L_{j}$ is the length of the filter. Note that the length of the filter can vary between different levels of decomposition denoted $\mathrm{j}$.

$$
\begin{aligned}
\tilde{W}_{j, t} & =\sum_{l=0}^{L_{j}-1} \tilde{h}_{j, l} r_{t-l \bmod N} \\
\tilde{V}_{j, t} & =\sum_{l=0}^{L_{j}-1} \tilde{g}_{j, l} r_{t-l \bmod N}
\end{aligned}
$$

As we incorporate the MODWT, the rescaled filters can be accessed from the DWT wavelet and scaling filters as in (2). Note that $J$ represents the total number of levels of decomposition, and $\mathrm{j}$ is the level of decomposition (Zhu et al., 2014).

$$
\tilde{h}_{j, l}=\frac{h_{j, l}}{2^{j / 2}}, \tilde{g}_{j, l}=\frac{g_{j, l}}{2^{j / 2}}, j=0, \ldots, J
$$

By classification, a DWT wavelet filter $h_{j, l}$ with length $L_{j}$ must satisfy the following properties (3).

$$
\sum_{l=0}^{L_{j}-1} h_{j, l}=0, \sum_{l=0}^{L_{j}-1} h_{j, l}^{2}=1, \sum_{l=0}^{L_{j}-1} h_{j, l} h_{j, l+2 n}=0, \forall n \in \mathbb{N}^{+}
$$

These properties ensure the elimination of redundant information in coefficients and resulting components, as "overlapping observations" are padded out, and only one "smooth" is used, i.e., the one corresponding to the largest scale. Moreover, energy preservation (unit energy/minimum variance) is achieved vis-a-vis the original series (Gençay et al., 2001; Percival \& Walden, 2000). The properties outlined in Eq. (3) are essential as they ensure that the set of wavelets constitutes an orthonormal basis, i.e., a signal can be decomposed using this set of wavelets. The DWT scaling filter is specified as the quadrature mirror of DWT wavelet filter satisfying conditions (4).

$$
h_{j, l}=(-1)^{l} g_{j, L_{j}-1-l}, \text { or }_{j, l}=(-1)^{l+1} h_{j, L_{j}-1-l}, l=0, \ldots, L_{j}-1 .
$$

The selection of the wavelet filter is crucial for deconstructing the underlying series to obtain the wavelet coefficients. We adopt the least asymmetric (LA) filters of Daubechies (1992) in MODWT to extract wavelet and scaling coefficients. LA filters are suitable to apprehend the scale and time differences in underlying series. In addition, the financial literature favors the utilization of $\operatorname{LA}(8)$ due to its ability to approximate phase linearity and near symmetric properties (Percival \& Walden, 2000). The linearity of phase indicates that the sinusoidal components and the events in the wavelet and scaling coefficients are aligned, across all levels of the decomposed series, to the original series. We determine the optimal MODWT decomposition level based on entropy specification criteria.

\subsection{Marginal distributions}

By Sklar's (1959) Theorem, a joint distribution of random variables can be expressed by their marginal distributions and the copula. Hence, selecting suitable marginal distributions for our 
time series is essential in estimating the copula. We chose the best-suited models for marginal distributions from different specifications, including GARCH, GJR-GARCH, and EGARCH for each decomposed series. Based on the AIC, the ARMA(1,0)-GJR-GARCH(1,1) specification is the best choice for capturing the dynamics of the underlying series. The GJR-GARCH is appropriate when negative and positive shocks tend to contribute asymmetrically to conditional volatility (Glosten et al., 1993). Accordingly, the mean-equations for return series $r_{t}$ are specified as in (5), where $\mu$ is the vector of constants, $\phi_{i}$ and $\theta_{j}$ are the autoregressive (AR) and moving average (MA) components with $m$ and $n$ lags, respectively.

$$
r_{t}=\mu+\sum_{i=1}^{m} \phi_{i} r_{t-i}+\varepsilon_{t}+\sum_{j=1}^{n} \theta_{j} \varepsilon_{t-j}
$$

The white noise process $\varepsilon_{t}$ follows a student-t distribution with $v$ degrees of freedom and the conditional variance, $\sigma_{t}^{2}$. Equation (6) shows the general form of the GJR-GARCH $(P, Q)$, where, $\Omega$ is the variance intercept, $\sigma_{\mathrm{t}-\mathrm{i}}^{2}$ the forecast error variance of the previous period (the GARCH component), $\varepsilon_{t-j}^{2}$ refer to past variances (the ARCH component), $\gamma_{j}$ represent the leverage effect, and $I$ is an indicator function taking the value $I[x<0]=1$ and zero otherwise.

$$
\sigma_{t}^{2}=\Omega+\sum_{i=1}^{P} \beta_{i} \sigma_{t-i}^{2}+\sum_{j=1}^{Q} \alpha_{j} \varepsilon_{t-j}^{2}+\sum_{j=1}^{Q} \gamma_{j} I\left[\varepsilon_{t-j}<0\right] \varepsilon_{t-j}^{2},
$$

For stationarity and positivity, $\Omega>0, \beta_{i} \geq 0,{ }_{j} \geq 0,{ }_{j}+\gamma_{j} \geq 0$, and $\sum_{i=1}^{P} \beta_{i}+$ $\sum_{j=1 j}^{Q}+1 / 2 \sum_{j=1}^{Q} \gamma_{j}<1$. The GJR model is suitable when negative shocks add more to the conditional volatility than positive shocks.

\subsection{Time-varying copula model}

We utilize the bivariate DCC-Student-t copula approach to assess the temporal dependence between different frequencies and tail dependence. This approach offers flexibility in accounting for extreme comovements, which are common in financial data. Using Sklar's (1959) theorem, we estimate the joint cumulative distribution function (cdf), $F_{X Y}(x, y)$, of two series, $X$ and $Y$, in terms of marginal cumulative distribution functions of each variable, $F_{X}(x)$ and $F_{Y}(y)$, and a copula function $C$ shown in (7).

$$
F_{X Y}(x, y)=C\left(F_{X}(x), F_{Y}(y)\right) .
$$

The joint probability density function (pdf), $f_{X Y}(x, y)$, can be estimated from the univariate marginal distributions of two underlying series, $f_{X}(x)$ and $f_{Y}(y)$, and the pdf of the copula distribution, $c(x, y)=\frac{\partial^{2} C(x, y)}{\partial x \partial y}$, as shown in (8). This follows from (7) after taking partial derivatives with respect to $\mathrm{x}$ and $\mathrm{y}$, assuming that all functions are differentiable.

$$
f_{X Y}(x, y)=c(x, y) f_{X}(x) f_{Y}(y) .
$$

We utilize the Student-t copula to evaluate extreme comovements in the series. Equation (9) expresses the multivariate case of a time-varying Student-t copula, where $d$ represents the degrees of freedom, $t_{d}^{-1}$ corresponds to the inverse univariate $t$ distribution, and $t_{d, \rho}$ characterizes the multivariate $t$ distribution with $\rho$ as the dependence matrix. Note that $\Gamma$ refers to the Gamma function. 


$$
\begin{aligned}
C_{d, \rho}\left(u_{1}, \ldots, u_{n}\right) & =t_{d, R}\left(t_{d}^{-1}\left(u_{1}\right), \ldots, t_{d}^{-1}\left(u_{n}\right)\right) \\
& =\int_{-\infty}^{t_{d}^{-1}\left(u_{1}\right)} \cdots \int_{-\infty}^{t_{d}^{-1}\left(u_{n}\right)} \frac{\Gamma\left(\frac{d+n}{2}\right)|\rho|^{-1 / 2}}{\Gamma\left(\frac{d}{2}\right)(\pi v)^{\frac{n}{2}}}\left(1+\frac{1}{d} z^{T} \rho^{-1} z\right)^{-\frac{d+n}{2}} \mathrm{~d} z_{1} \cdots \mathrm{d} z_{n}
\end{aligned}
$$

We consider the temporal dependence among the underlying assets by replacing the linear correlation $\rho$ with the dynamic conditional correlation (DCC) coefficient of Engle (2002). Consequently, the time-varying DCC matrix, $R_{t}$, is given by (10), where $\Omega=(1--\beta) \bar{R}$, and $\beta$ are always positive with their sum being less than 1 , and $\epsilon_{t}$ represents the standardized returns stemming from the ARMA(1,0)-GJR-GARCH(1,1) specification. The latter is obtained from fitting models for marginal distributions as outlined in Sects. 2.2 and 4.1.

$$
R_{t}=\operatorname{diag}\left(\tilde{Q}_{t}\right)^{-1} Q_{t} \operatorname{diag}\left(\tilde{Q}_{t}\right)^{-1}, Q_{t}=\Omega+\alpha \epsilon_{t-1} \epsilon_{t-1}^{\prime}+\beta Q_{t-1},
$$

\subsection{Estimation process}

The copula parameters are estimated by utilizing a two-step maximum likelihood technique. The inference functions for margins (IFM) method is based on Joe (1997). The first step of this procedure comprises the estimation of univariate GARCH parameters, $\widehat{\theta}_{1}$ related to the marginal distributions as in (11).

$$
\hat{\theta}_{1}=\operatorname{argmax}_{\theta_{1}} \sum_{t=1}^{T} \sum_{j=1}^{n} \ln f_{j}\left(u_{j t} ; \theta_{1}\right) .
$$

Based on $\widehat{\theta}_{1}$, we estimate DCC-student-t copula parameter, $\widehat{\theta}_{2}$, in the second step (12).

$$
\hat{\theta}_{2}=\operatorname{argmax}_{\theta_{2}} \sum_{t=1}^{T} \ln c\left(F_{1}\left(u_{1 t}\right), F_{2}\left(u_{2 t}\right), \ldots, F_{n}\left(u_{n t}\right) ; \theta_{2}, \hat{\theta}_{1}\right) .
$$

The interested readers are referred to Joe (1997) and Patton (2006) for a detailed discussion of copulas.

\section{Data and descriptive analysis}

\subsection{Sampling and variables}

We collected daily data from January 2, 1995 to February 7, 2019 of biotech indices, including the Nasdaq biotechnology index (Nasdaq biotech), the Dow Jones U.S. Biotechnology Index (DJUSBT US biotech), and the S\&P 500 pharmaceuticals index (SP500 pharma). Other asset classes refer to the NYSE ARCA Tech index (NYSE ARCA)), commodities (crude oil and gold), the equity markets of the United States, the UK, and Europe (S\&P 500, FTSE 100, and Stoxx600E), and the Euro to the USD exchange rate.

The Nasdaq biotechnology index comprises 278 companies listed on the NASDAQ. The top five constituents Amgen, Gilead, Illumina, Vertex, and Moderna, account for about 30\% of the index. In contrast, the Dow Jones U.S. Biotechnology index only represents 17 companies, including the top four companies in the Nasdaq biotechnology index. The S\&P 500 
pharmaceuticals index differs in that it uses an equal-weighted market cap method. Hence, it is less skewed towards large corporations. Moreover, the index is based on 49 constituents, representing a larger base than the Dow Jones U.S. Biotechnology index. The other asset classes refer to mostly standard indices. Note that the STOXX Europe 600 is a broad index, which also contains some UK-listed firms; however, this overlap only refers to about $20 \%$ of the index. The selected data is extracted from DataStream International. The investigation period covers the Asian financial crisis (1997/1998), the dot.com bubble (2001), the Iraq war (2003), the GFC, the ESDC, and the deterioration of crude oil prices in 2014.

\subsection{Descriptive statistics}

For all series, we calculate log-returns defined as $r_{i, t}=\ln \left(P_{i, t} / P_{i, t-1}\right)$. Table 1 reports descriptive statistics, including annualized means, the annualized standard deviation, and Sharpe ratios (SR). Sharpe ratios, i.e., the reward-to-risk measure, indicate that biotech and pharmaceutical indices significantly outperform. ${ }^{2}$ Return distributions for nearly all the assets are negatively skewed. The values of kurtosis are higher than 3 in all cases. Hence, the return distributions are skewed negatively and exhibit leptokurtic distributions, indicating asymmetric and fat tails. The null hypothesis of normality is strongly rejected by the Jarque-Bera test as expected. The Ljung-Box test-statistics with 15 lags for returns and squared returns are significant at the $1 \%$ threshold level, indicating serial correlation in both series. The test statistics from ARCH-LM with 15 lags strongly reject the null of conditional homoscedasticity, suggesting the utilization of a GARCH-type specification to model the volatility clustering and temporal dynamics.

We use a Symlet wavelet and derive the optimal level of decomposition, which is eight in all cases. The MODWT decomposes the original time series based on eight scales. The first scale refers to a high-frequency signal, which corresponds to a short period. One could interpret the first scale as the short-run horizon of investors. Higher scales could be interpreted as a medium to long-term horizons as they refer to longer periods. Figure 1 illustrates the decomposition of return series into short-, medium-, and long-run components based on the Nasdaq Biotech Index. The decomposed series becomes less volatile at lower frequency levels, i.e., higher scales.

\section{Empirical analysis}

\subsection{Fitting models for marginal distribution}

The DCC-Student-t copula parameters are estimated by utilizing a two-step procedure. In the first step, we estimate marginal distributions models as outlined in Sect. 2.2, i.e., univariate GARCH-type models for undecomposed and decomposed series. Table 2 presents the ARMA(1,0)-GJR-GARCH(1,1) specification parameters for the undecomposed series. In mean-equations, the first-order autocorrelation coefficient $(A R(1))$ is insignificant-except for gold price changes. This indicates limited predictability of returns due to highly efficient markets. The parameters $(\alpha)$ and $(\beta)$, representing $\mathrm{ARCH}$ and lagged conditional variance, are strongly significant at the $1 \%$ significance level suggesting that current variance is affected by lagged squared shocks and persistency in conditional volatility.

2 The risk-free rate in reward-to-risk measure is T-bill rate, which is estimated to be around $1 \%$. 


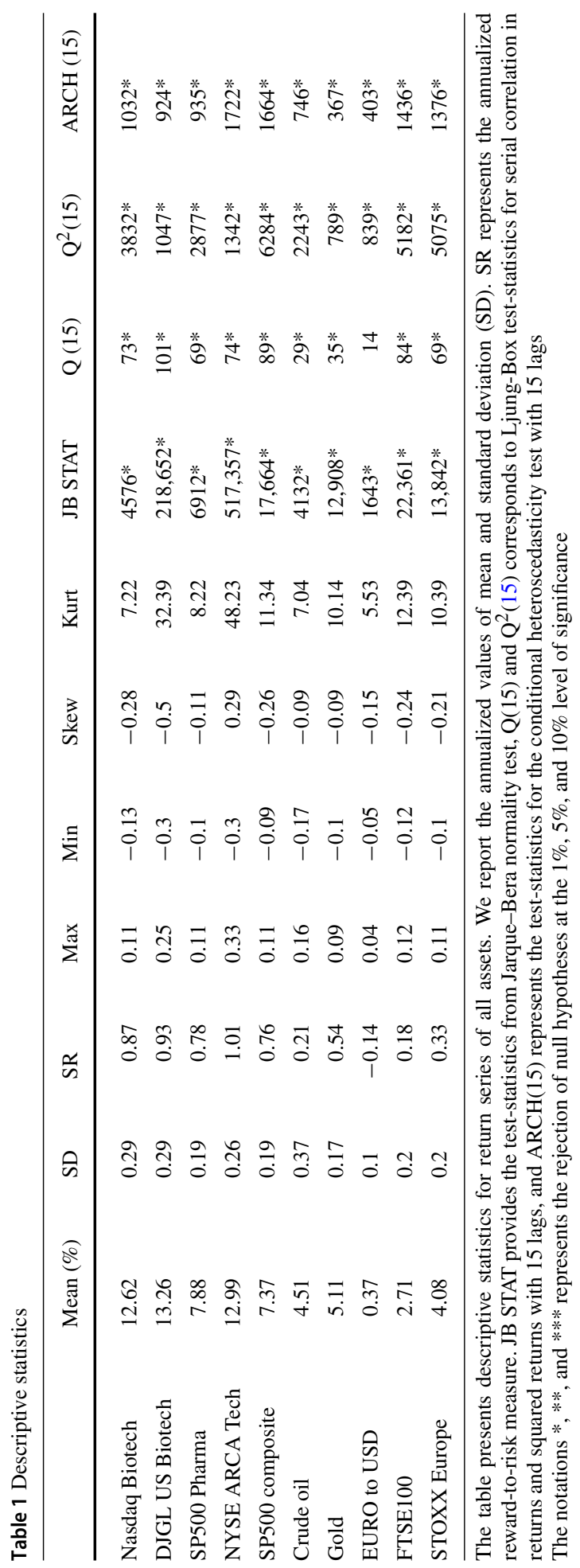




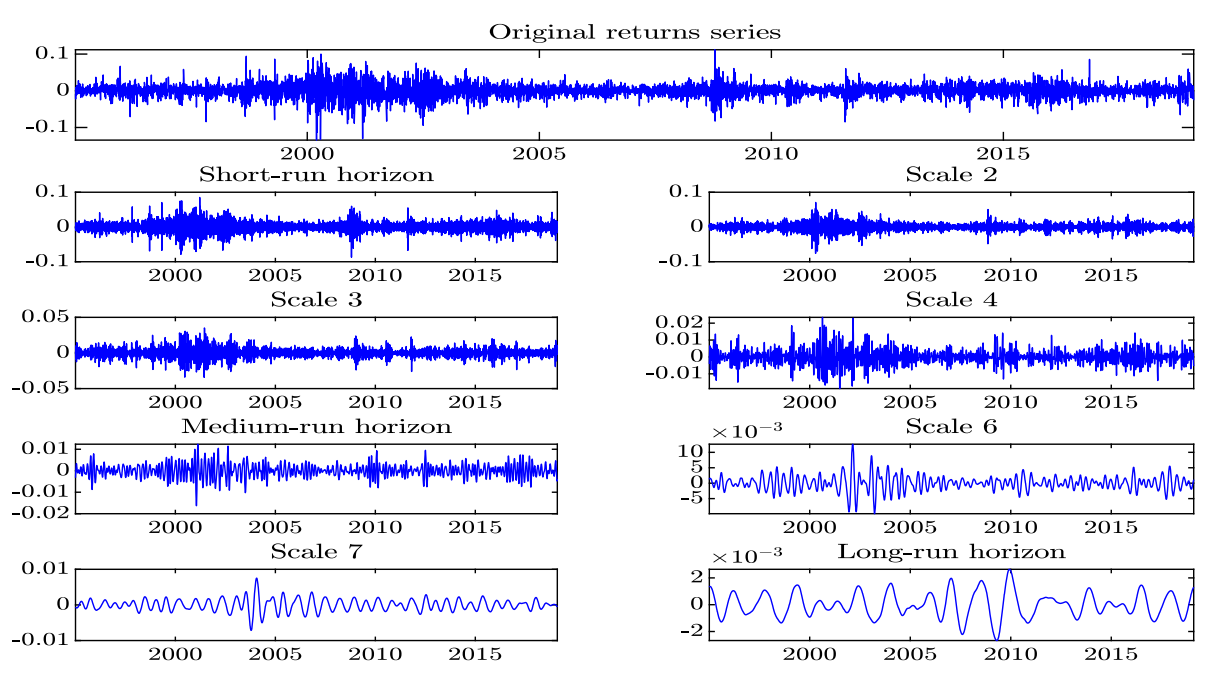

Fig. 1 The return of the Nasdaq Biotech Index was decomposed using a Symlet wavelet. The optimal level of decomposition is eight. Scale 1 refers to the highest frequency and shortest period, whereas scale 8 represents the lowest frequency and longest period. We interpret the first scale as the short-run, scale 5 as the medium, and scale 8 as the long-term horizon

The parameter capturing the asymmetric impact of good and bad news on conditional volatility $(\gamma)$ is strongly significant at the $1 \%$ significance level. Furthermore, the degrees of freedom parameter (DoF) is strongly significant, with values exceeding two for all assets indicating a potential for tail dependence. Hence, extreme joint movements and fat tails characterize all distributions. Diagnostics suggest that an $\operatorname{ARMA}(1,0)-\mathrm{GJR}-\mathrm{GARCH}(1,1)$ specification together with a Student-t distribution is adequate for modeling stylized facts of all the assets in our sample. Although the deviation from normality still exists in residuals, there is no autocorrelation and ARCH effects remaining in nearly all assets, suggesting stability of the employed models for marginal distributions.

\subsection{Estimates of copula functions}

Based on GJR-GARCH filtered returns, we evaluate the connectedness dynamics between the biotech indices and other asset classes by employing a DCC-Student-t copula framework. Table 3 presents the copula estimates for original and decomposed returns series between the Nasdaq biotech and other underlying assets. The connectedness parameter is strongly significant at the $1 \%$ threshold level for all assets. The dependence parameter is high between the Nasdaq biotech and other biotech assets over both undecomposed and decomposed series.

Furthermore, the connectedness structure between the Nasdaq biotech and other financial indices may also be characterized as moderate to strong dependence across all series. This shows that biotech indices and financial assets tend to move together. In contrast, the linkage structure between the Nasdaq biotech and commodities or exchange rates is weak and negative, indicating diversification benefits over various frequency horizons. The parameters capturing the asymmetric effect of shocks $(\alpha)$ and persistency in conditional connectedness $(\beta)$ are strongly significant at the $1 \%$ significance level. The degrees of freedom (DoF) parameter is low and strongly significant at the $1 \%$ significance level for the undecomposed series 


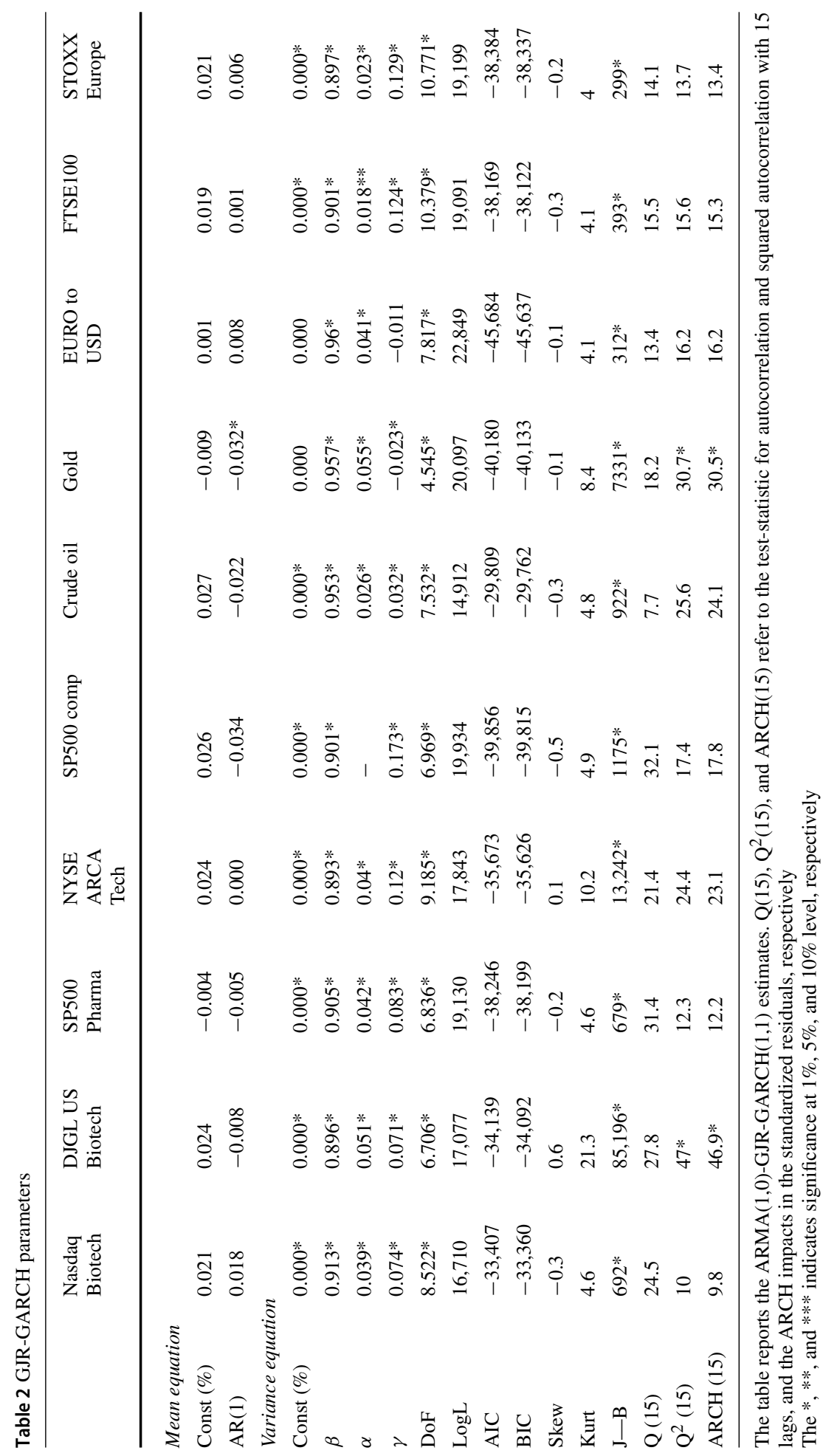




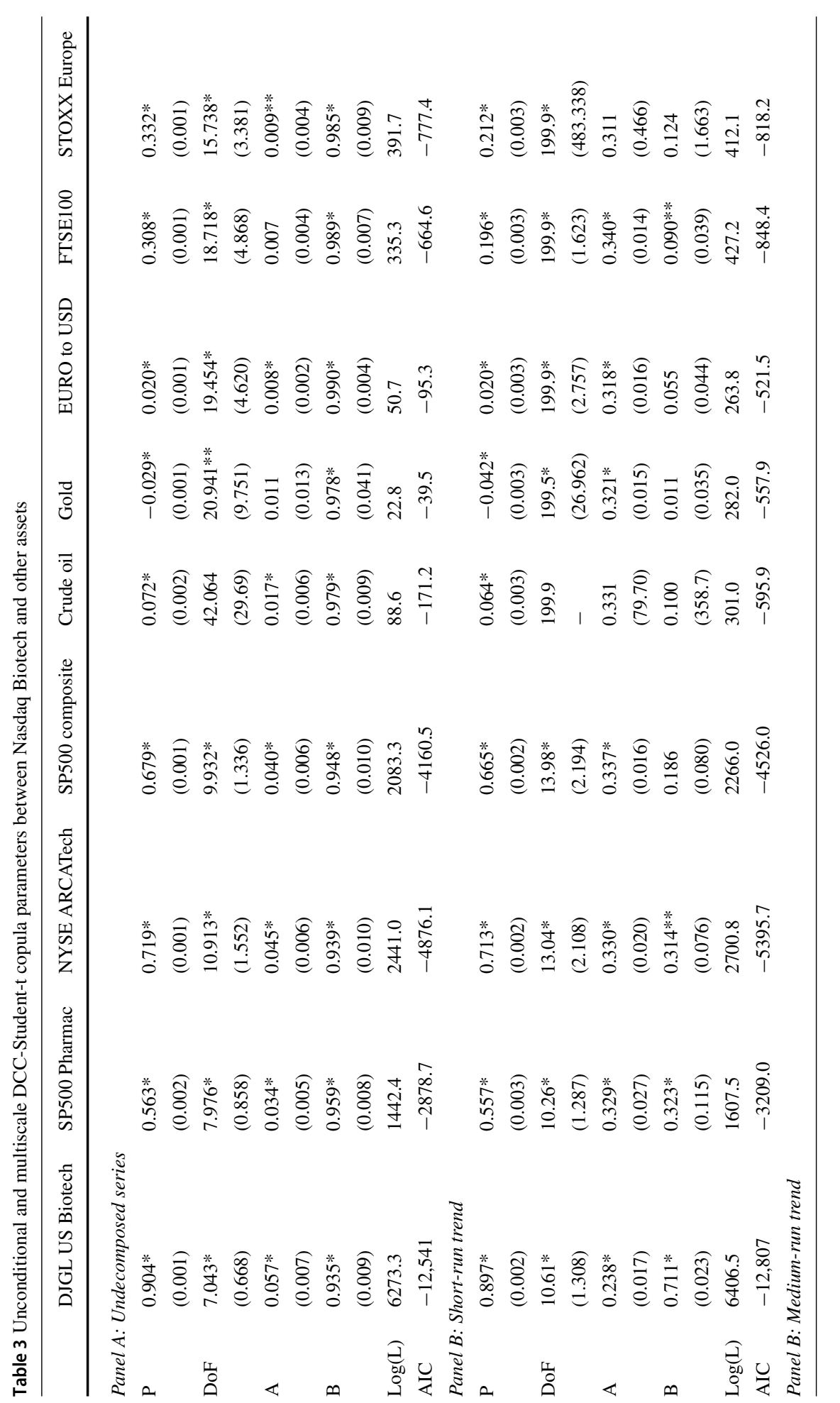




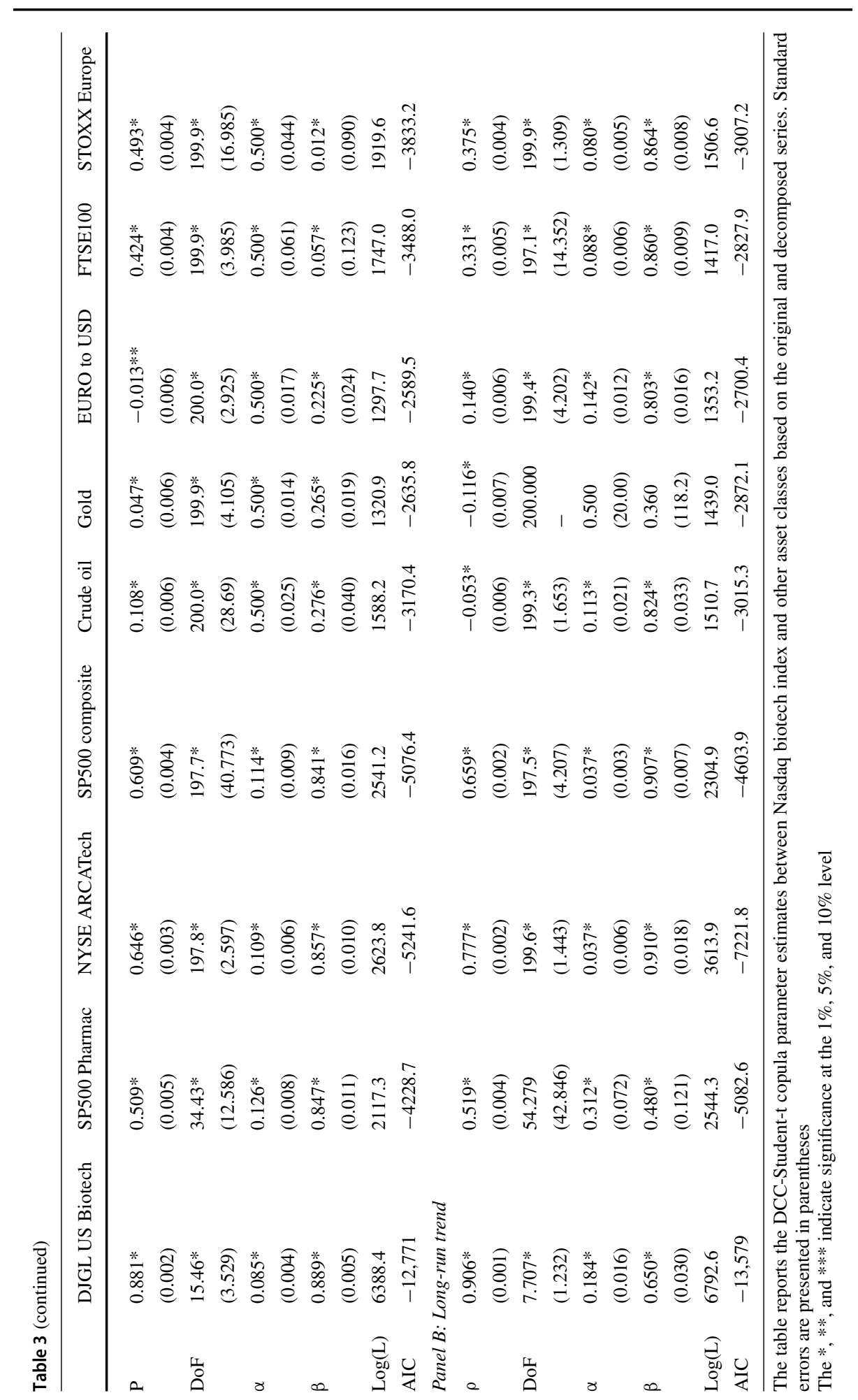


and the short-run trend. However, the DoF parameter is large for lower frequencies indicating that over those frequencies, the return distribution converges to standard normal distribution. The lower and significant estimates of DoF show that the extreme joint movement is highly likely for the undecomposed and short-run trend. Although the dependence parameter does not change considerably for biotech indices and the three underlying financial indices, the direction and intensity of connectedness vary in the case of crude oil, gold, and the Euro to the USD exchange rate. It is noteworthy that the dependence of the Nasdaq biotech index with crude oil and gold differs between the undecomposed series $(0.072$ and -0.029$)$ and the long-run horizon $(-0.053$ and -0.116$)$, respectively. However, the connectedness dynamics between the Nasdaq biotech index and the Euro to USD exchange rate closely follow the neutrality over the original, short-, and medium-run series. This indicates a strong diversification potential over the long run. Similar diversification and risk management benefits may be achieved by utilizing the Nasdaq biotech index and the Euro to the USD exchange rate in a portfolio over the original, short-, and medium-run series.

Additional analyses were conducted focusing on the DJGL US biotech, S\&P500 pharmaceuticals, and the NYSE ARCA Tech indices, which lead to qualitatively similar findings. In summary, there are pronounced diversification benefits when combining investment in biotech with foreign exchange markets and commodities. The following section conducts portfolio analyses to investigate these alleged diversification benefits.

\section{Portfolio analyses}

\subsection{Value-at-risk, CoVaR, and $\Delta$ CoVaR}

Value-at-risk ( $\mathrm{VaR})$ is the statistical measure of possible losses of investment in an asset, where losses greater than the $\mathrm{VaR}$ are suffered with a specified probability. We quantify the downside risk of investment in biotech and other asset classes. Let $r_{t}$ be the continuously compounded return of the underlying asset then the $\operatorname{Va} R_{1, t}^{\alpha}$ is the $\alpha^{\text {th }}$ quantile of the return distribution defined in Eq. (13).

$$
\operatorname{Pr}\left(r_{1, t} \leq \operatorname{VaR} R_{1, t}^{\alpha}\right)=\alpha
$$

The downside CoVaR is defined as the VaR for an underlying asset conditional on extreme outcomes of another asset. Given a bivariate time series $r_{t}=\left(r_{1, t}, r_{2, t}\right): t=1,2, \ldots, N$ with $r_{2, t}$ representing each of the biotech assets and $r_{1, t}$ representing other underlying assets, ${ }^{3}$ the downside CoVaR of biotech returns conditional on the extreme downward movements of other assets, which is expressed in (14), where $\operatorname{Pr}\left(r_{2, t} \leq \operatorname{VaR} R_{2, t}^{\beta}\right)=\beta$ with $\beta$ as the tail probability distribution of the other assets.

$$
\operatorname{Pr}\left(r_{1, t} \leq \operatorname{CoVaR} R_{(1 \mid 2, t)}^{\alpha} \mid r_{2, t} \leq \operatorname{VaR}_{2, t}^{\beta}\right)=\alpha
$$

In addition to the VaR and CoVaR, we estimate the Delta CoVaRs ( $\Delta$ CoVaR), which can be interpreted as the difference between the VaR for other underlying assets conditional on an extreme movement of biotech assets and the $\mathrm{VaR}$ of the underlying asset returns conditional on the normal state (median values) of the biotech assets, which can be expressed in (15).

3 Other underlying assets corresponds to biotech indices, financial market indices, commodities, and exchange rates. 


$$
\begin{gathered}
\operatorname{CoVaR}_{1 \mid 2 M, t}^{\alpha} \text { satisfy that } \operatorname{Pr}\left(r_{1, t} \leq \operatorname{CoVaR}_{1 \mid 2 M, t}^{\alpha} \mid F_{(2, t)\left(r_{2}, t\right)}=0.5\right)=\alpha \\
\Delta \operatorname{CoVaR} R_{1 \mid 2, t}^{\alpha}=\left(\operatorname{CoVaR}_{1 \mid 2, t}^{\alpha}-\operatorname{CoVaR}_{1 \mid 2 M, t}^{\alpha}\right)
\end{gathered}
$$

Table 4 provides the estimates of VaR of all undecomposed and decomposed return series. Based on VaR estimates, the Euro to USD exchange rate exhibits the lowest risk as abrupt changes are rather uncommon. This is followed by gold and the S\&P500 composite, exhibiting $-1.598 \%$ and $-1.792 \%$, respectively. The highest estimate of VaR occurs in crude oil, exhibiting a value of $-3.782 \%$, which may be due to its sensitivity to financial, economic, and political uncertainties. In the case of biotech assets, the S\&P500 pharmaceuticals index provides the lowest values of $\mathrm{VaR}$ of $-1.854 \%$. However, it is noteworthy that the VaR estimates tend to decline with longer periods (low frequency), which may be attributed to the lower volatility of asset returns over the long term (Carvalho et al., 2018).

Table 5 shows our results of CoVaRs between the Nasdaq biotech index and other asset classes. The descriptive statistics of CoVaR indicate that the downside VaR and CoVaR exhibit similar trends in all cases, with only minor differences in magnitude. Furthermore, analogous to long-run estimates of $\mathrm{VaR}$, the values of CoVaR also decline with lower frequencies.

Regarding $\triangle \mathrm{CoVaR}$, the results indicate that the values are different from the CoVaR estimates demonstrating extreme uncertainty to risk relative to a normal state (i.e., median values) as shown in Table 6. However, considering lower frequency dimensions, the values of $\triangle \mathrm{CoVaR}$ for the underlying assets conditional on biotech assets approach zero. This indicates that, over the long run, there is no difference between the CoVaRs for underlying asset returns between normal conditions and extreme outcomes in the biotech sector.

\subsection{Portfolio designs and hedging}

In addition to our VaR and CoVaRs analyses, we estimate temporal and frequential optimal portfolio weights and hedge ratios between biotech assets and other underlying assets. Following Kroner and $\mathrm{Ng}$ (1998), we derive risk-minimizing weights for portfolios consisting of a biotech and a non-biotech asset. This binary approach is common when comparing asset classes. As outlined in Kroner and Sultan (1993), a mean-variance optimizing investor maximizes her expected utility, which consists of the expected return minus her coefficient of risk aversion times the variance. This can be derived from a CARA (constant absolute risk aversion) utility function taking logs, i.e., applying a positive monotonic transformation. Hence, portfolio weights are a function of risk preferences, which limits their use if these preferences are unknown or heterogeneous among a group of investors. Kroner and Sultan (1993) assume that futures prices are martingales, i.e., $E_{t}\left(P_{t+1}\right)=P_{t}$. This assumption implies that future expected returns are zero as stated in Kroner and $\mathrm{Ng}$ (1998). Based on this assumption, simplified hedge ratio that do not depend on the degree of risk aversion can be derived.

Our methodology focuses on estimating the time-varying covariance matrix, which is the driver of portfolio weights as outlined in Kroner and $\mathrm{Ng}$ (1998). As can be seen in Table 2 , the mean-equation predicts expected returns close to zero, which is expected in the case of efficient markets. Hence, the assumption that future expected returns are zero cannot be rejected empirically.

Optimal weights of the other underlying assets (OA) denoted $w_{t}^{O A, B A}$ are derived in (16) at time $t$. The conditional variance of biotech assets and other underlying assets refer to $h_{t}^{B A}$ and $h_{t}^{O A}$ respectively, and $h_{t}^{O A, B A}$ is the conditional covariance of biotech assets with other 


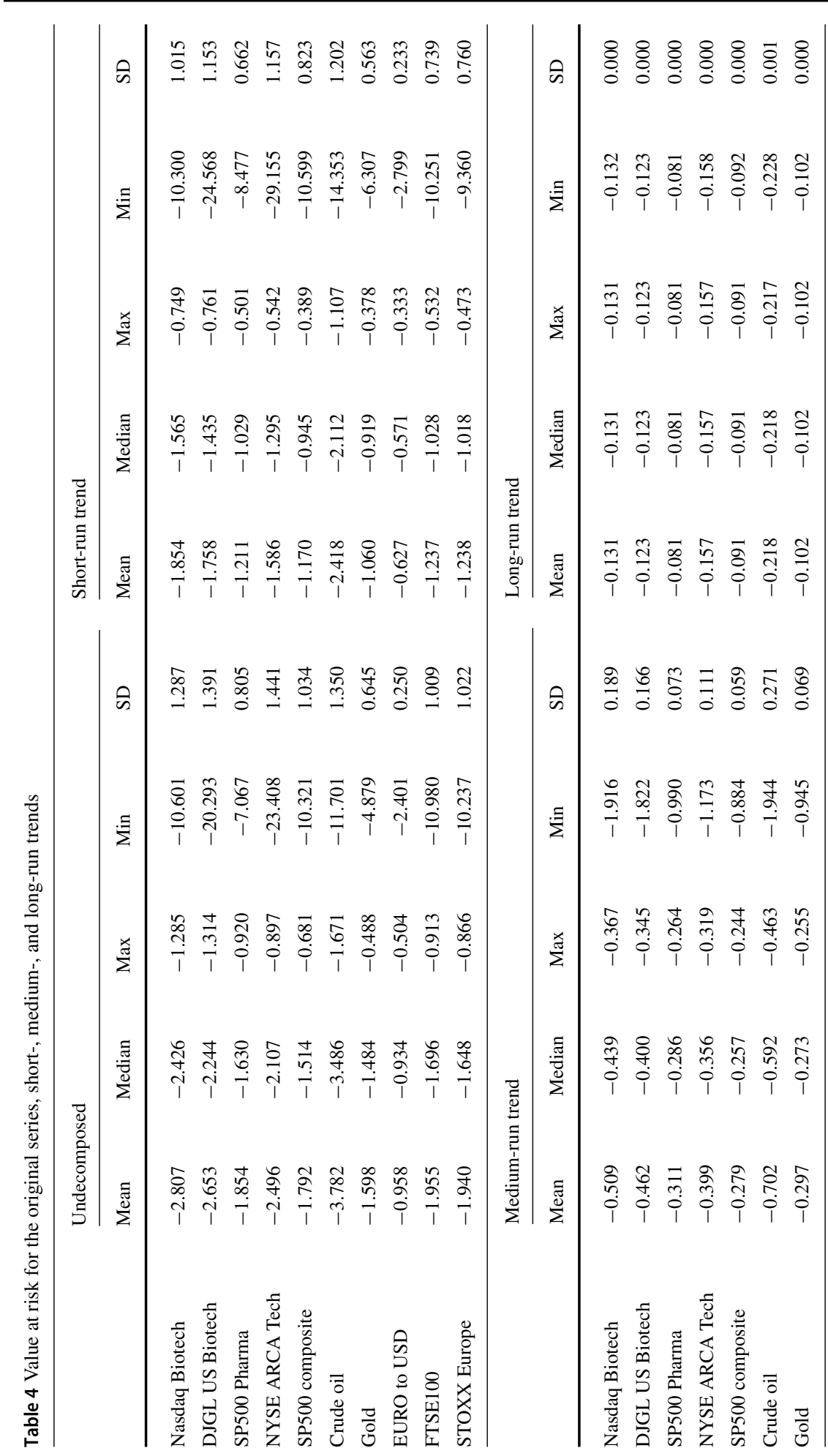




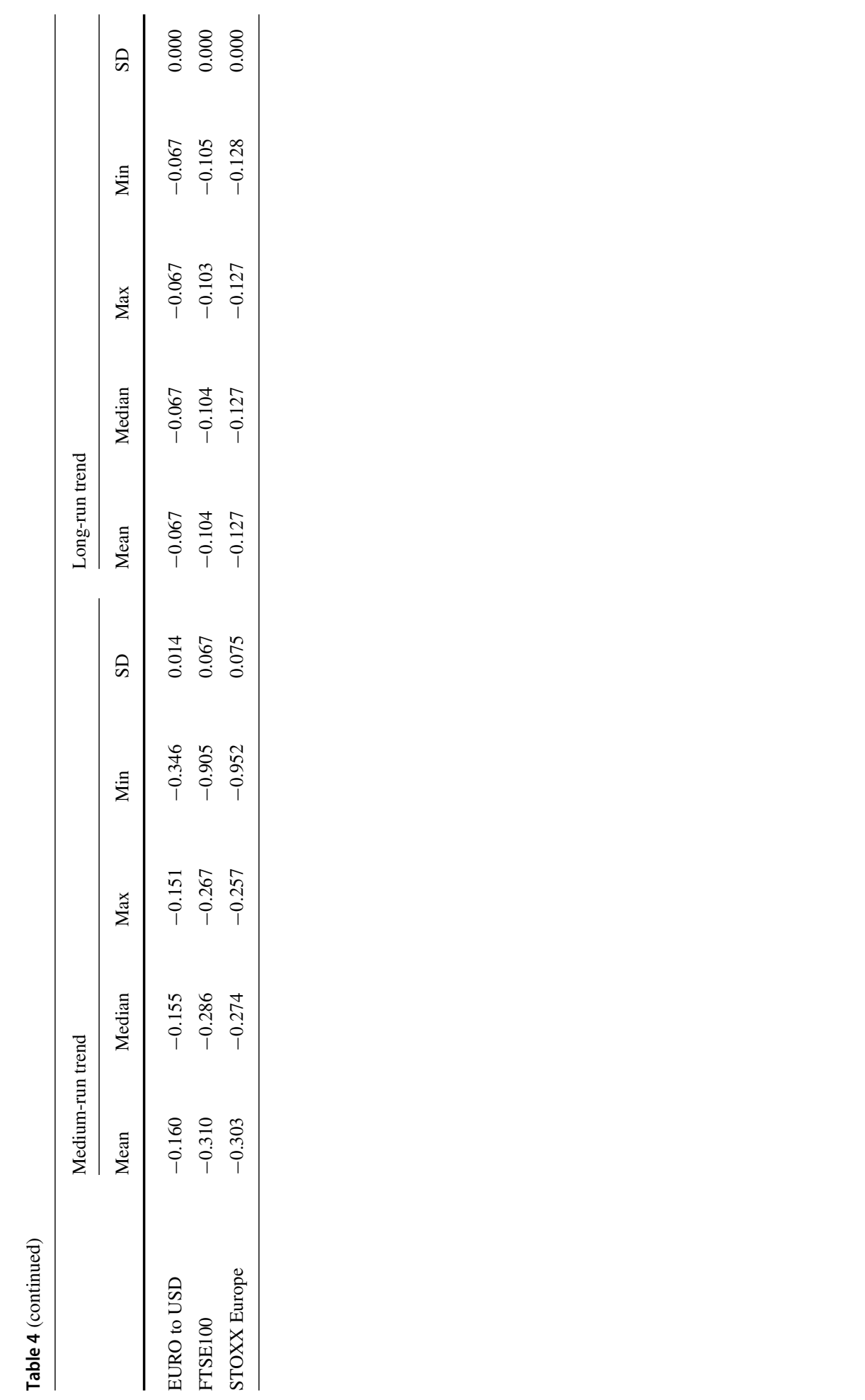




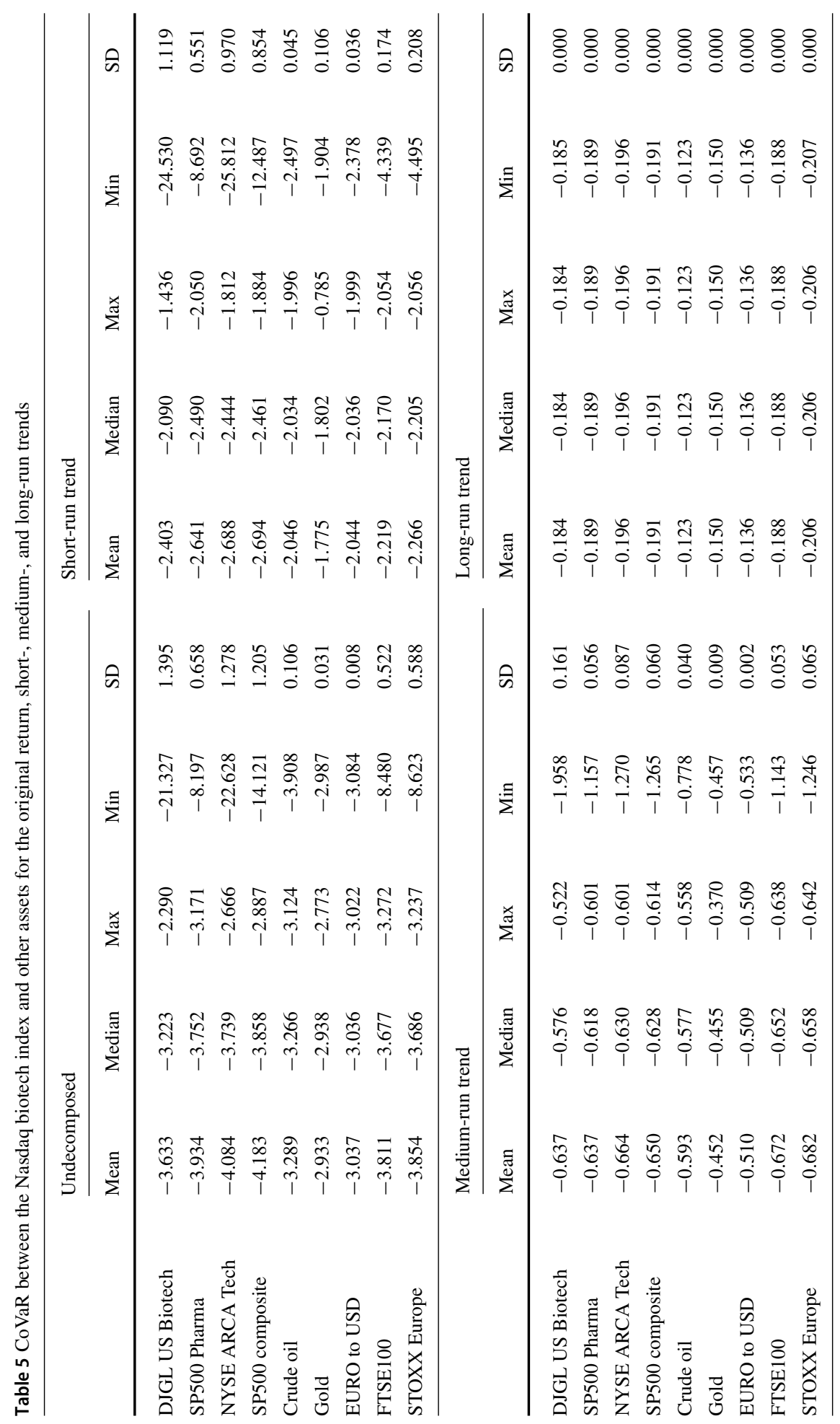




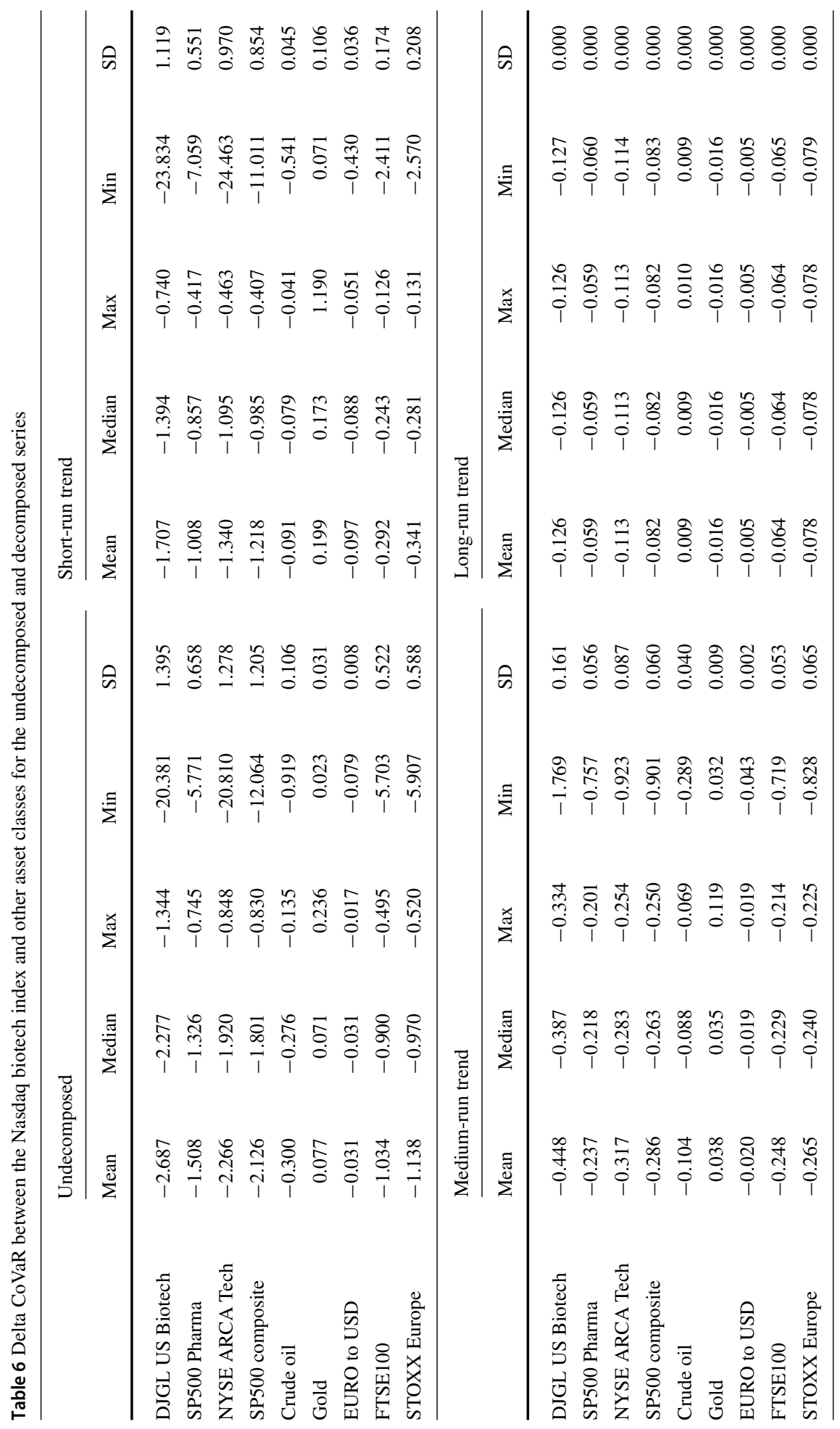


assets. Note that conditional variances and the covariance are conditional with respect to time, i.e., the information set at time t. In line with Kroner and $\mathrm{Ng}$ (1998), portfolios must be fully investing, and short selling is not permitted. Equation (16) applies these conditions and derives $\tilde{w}_{t}^{O A, B A}$. The weight of biotech assets in the bivariate portfolio is estimated as $1-w_{t}^{O A, B A}$.

$$
\begin{gathered}
w_{t}^{O A, B A}=\frac{h_{t}^{B A}-h_{t}^{O A, B A}}{h_{t}^{O A}-2 h_{t}^{O A, B A}+h_{t}^{B A}} \\
\tilde{w}_{t}^{O A, B A}= \begin{cases}0 & \text { if } w_{t}^{O A, B A}<0 \\
w_{t}^{O A, B A} & \text { if } 0 \leq w_{t}^{O A, B A} \leq 1 \\
1 & \text { if } w_{t}^{O A, B A}>1\end{cases}
\end{gathered}
$$

This binary approach is common when comparing asset classes. For instance, Arouri et al. (2011) adopted the same methodology to derive portfolios based on equities (using stock market indices) and oil prices. Using a multivariate approach requires making further assumptions such as the optimal number of asset classes, risk preferences, liquidity needs, and many other factors, which go beyond the scope of the paper.

Table 7 presents an overview of portfolio weights for undecomposed and decomposed series estimated from our ARMA(1,0)-GJR-GARCH(1,1) specification and a DCC-Studentt copula. Our analysis indicates that the average portfolio weight of the Euro to USD/Nasdaq biotech portfolio is 0.868 , suggesting that for $\$ 1$ portfolio, 86.8 cents should be allocated in the Euro to USD exchange rate and $13.2(1-0.868)$ cents should be invested in the Nasdaq biotech index. However, with the longer investment horizon, portfolio weights converge to equally weighted portfolios.

In addition, following Kroner and Sultan (1993), we estimate hedge ratios for bivariate portfolios, i.e., each of biotech asset and other asset classes. To minimize the uncertainty of the portfolio that is $\$ 1$ invested in other assets, the investor should short $\$ \beta$ of biotech assets. We estimate the hedge ratios as in (17).

$$
\beta_{t}^{O A, B A}=\frac{h_{t}^{O A, B A}}{h_{t}^{B A}}
$$

Hedge ratios are significantly higher when considering a biotech index and other financial indices. For instance, the hedge ratio between the S\&P500 composite/the Nasdaq biotech index is 0.436 , which indicates that a $\$ 1$ long position in the S\&P500 composite index can be hedged with a 43.6 cents short position in the Nasdaq biotech index. However, it is noteworthy that the cost of hedging is significantly lower in the case of bivariate portfolios consisting of biotech assets with commodities and exchange rate, which is in line with VaR estimates. For instance, in the case of the Nasdaq biotech index and the Euro to the USD exchange rate, a $\$ 1$ long position in the Euro to USD exchange rate can be hedged with a 0.2 cents position in the Nasdaq biotech index. Similarly, in the case of the NYSE ARCA Tech index and gold, a \$1 long position in gold can be hedged with a 1.8 cents long position in NYSE ARCA Tech. Overall, our findings indicate a significant potential to realize diversification and risk management benefits by combining biotech assets with other commodities or exchange rates.

\section{Conclusions}

This study evaluates the temporal and spectral interdependence among biotech assets and other asset classes by employing wavelet-based DCC-Student-t copulas. Specifically, we 
Table 7 Summary statistics of portfolio weights and hedge ratios (HR)

\begin{tabular}{|c|c|c|c|c|c|c|c|c|}
\hline & \multicolumn{2}{|l|}{ Returns } & \multicolumn{2}{|c|}{ Short-run trend } & \multicolumn{2}{|c|}{ Medium-run trend } & \multicolumn{2}{|c|}{ Long-run trend } \\
\hline & Weight & HR & Weight & HR & Weight & HR & Weight & HR \\
\hline \multicolumn{9}{|c|}{ Panel A: Nasdaq Biotech } \\
\hline $\begin{array}{c}\text { DJGL US } \\
\text { Biotech }\end{array}$ & 0.696 & 0.863 & 0.673 & 0.854 & 0.576 & 0.862 & 0.502 & 0.906 \\
\hline $\begin{array}{l}\text { SP500 } \\
\text { Pharma }\end{array}$ & 0.838 & 0.396 & 0.817 & 0.391 & 0.621 & 0.466 & 0.501 & 0.518 \\
\hline $\begin{array}{l}\text { NYSE ARCA } \\
\text { Tech }\end{array}$ & 0.731 & 0.621 & 0.697 & 0.629 & 0.593 & 0.617 & 0.500 & 0.777 \\
\hline SP500 comp & 0.904 & 0.436 & 0.892 & 0.409 & 0.656 & 0.541 & 0.501 & 0.659 \\
\hline Crude oil & 0.355 & 0.098 & 0.367 & 0.094 & 0.462 & 0.114 & 0.498 & -0.053 \\
\hline Gold & 0.697 & -0.014 & 0.700 & -0.024 & 0.572 & 0.046 & 0.500 & -0.116 \\
\hline $\begin{array}{l}\text { EURO to } \\
\text { USD }\end{array}$ & 0.868 & 0.002 & 0.861 & 0.007 & 0.613 & -0.013 & 0.500 & 0.140 \\
\hline FTSE100 & 0.717 & 0.232 & 0.692 & 0.153 & 0.602 & 0.387 & 0.500 & 0.331 \\
\hline $\begin{array}{l}\text { STOXX } \\
\text { Europe }\end{array}$ & 0.723 & 0.249 & 0.695 & 0.166 & 0.597 & 0.455 & 0.500 & 0.375 \\
\hline \multicolumn{9}{|c|}{ Panel B: DJGL US Biotech } \\
\hline $\begin{array}{l}\text { Nasdaq } \\
\text { Biotech }\end{array}$ & 0.304 & 0.963 & 0.327 & 0.972 & 0.424 & 0.920 & 0.498 & 0.906 \\
\hline $\begin{array}{l}\text { SP500 } \\
\text { Pharma }\end{array}$ & 0.807 & 0.421 & 0.781 & 0.421 & 0.590 & 0.521 & 0.501 & 0.522 \\
\hline $\begin{array}{l}\text { NYSE ARCA } \\
\text { Tech }\end{array}$ & 0.649 & 0.615 & 0.628 & 0.635 & 0.562 & 0.539 & 0.499 & 0.672 \\
\hline SP500 comp & 0.875 & 0.441 & 0.860 & 0.422 & 0.617 & 0.523 & 0.500 & 0.620 \\
\hline Crude oil & 0.334 & 0.087 & 0.341 & 0.074 & 0.450 & 0.081 & 0.498 & -0.048 \\
\hline Gold & 0.679 & -0.025 & 0.678 & -0.032 & 0.557 & 0.046 & 0.500 & -0.151 \\
\hline $\begin{array}{l}\text { EURO to } \\
\text { USD }\end{array}$ & 0.855 & 0.002 & 0.847 & 0.006 & 0.599 & -0.034 & 0.500 & 0.196 \\
\hline FTSE100 & 0.684 & 0.220 & 0.658 & 0.142 & 0.581 & 0.362 & 0.499 & 0.248 \\
\hline $\begin{array}{l}\text { STOXX } \\
\text { Europe }\end{array}$ & 0.692 & 0.239 & 0.662 & 0.158 & 0.575 & 0.416 & 0.500 & 0.337 \\
\hline \multicolumn{9}{|c|}{ Panel C: SP500 Pharma } \\
\hline $\begin{array}{l}\text { Nasdaq } \\
\text { Biotech }\end{array}$ & 0.162 & 0.854 & 0.183 & 0.891 & 0.379 & 0.592 & 0.499 & 0.519 \\
\hline $\begin{array}{r}\text { DJGL US } \\
\text { Biotech }\end{array}$ & 0.193 & 0.818 & 0.219 & 0.849 & 0.410 & 0.637 & 0.499 & 0.523 \\
\hline $\begin{array}{l}\text { NYSE ARCA } \\
\text { Tech }\end{array}$ & 0.324 & 0.611 & 0.327 & 0.684 & 0.445 & 0.442 & 0.499 & 0.313 \\
\hline SP500 comp & 0.625 & 0.611 & 0.649 & 0.606 & 0.532 & 0.634 & 0.500 & 0.437 \\
\hline Crude oil & 0.209 & 0.058 & 0.218 & 0.055 & 0.403 & -0.044 & 0.497 & 0.029 \\
\hline Gold & 0.546 & -0.047 & 0.546 & -0.043 & 0.503 & 0.015 & 0.500 & -0.123 \\
\hline
\end{tabular}


Table 7 (continued)

\begin{tabular}{|c|c|c|c|c|c|c|c|c|}
\hline & \multicolumn{2}{|l|}{ Returns } & \multicolumn{2}{|c|}{ Short-run trend } & \multicolumn{2}{|c|}{ Medium-run trend } & \multicolumn{2}{|c|}{ Long-run trend } \\
\hline & Weight & HR & Weight & HR & Weight & HR & Weight & HR \\
\hline $\begin{array}{l}\text { EURO to } \\
\text { USD }\end{array}$ & 0.756 & -0.017 & 0.739 & -0.009 & 0.553 & -0.044 & 0.500 & 0.025 \\
\hline FTSE100 & 0.480 & 0.352 & 0.487 & 0.225 & 0.506 & 0.490 & 0.499 & 0.322 \\
\hline $\begin{array}{l}\text { STOXX } \\
\text { Europe }\end{array}$ & 0.487 & 0.375 & 0.491 & 0.249 & 0.498 & 0.497 & 0.499 & 0.273 \\
\hline \multicolumn{9}{|c|}{ Panel D: NYSE ARCA Tech } \\
\hline $\begin{array}{l}\text { Nasdaq } \\
\text { Biotech }\end{array}$ & 0.269 & 0.902 & 0.303 & 0.912 & 0.407 & 0.731 & 0.500 & 0.777 \\
\hline $\begin{array}{c}\text { DJGL US } \\
\text { Biotech }\end{array}$ & 0.351 & 0.806 & 0.372 & 0.815 & 0.438 & 0.610 & 0.501 & 0.672 \\
\hline $\begin{array}{l}\text { SP500 } \\
\text { Pharma }\end{array}$ & 0.676 & 0.435 & 0.673 & 0.454 & 0.555 & 0.391 & 0.501 & 0.313 \\
\hline SP500 comp & 0.895 & 0.645 & 0.892 & 0.606 & 0.651 & 0.782 & 0.502 & 0.866 \\
\hline Crude oil & 0.269 & 0.196 & 0.286 & 0.178 & 0.412 & 0.190 & 0.494 & 0.132 \\
\hline Gold & 0.621 & -0.018 & 0.625 & -0.034 & 0.530 & 0.021 & 0.500 & 0.061 \\
\hline $\begin{array}{l}\text { EURO to } \\
\text { USD }\end{array}$ & 0.817 & 0.000 & 0.807 & 0.009 & 0.581 & -0.015 & 0.500 & -0.096 \\
\hline FTSE100 & 0.610 & 0.366 & 0.601 & 0.238 & 0.553 & 0.526 & 0.500 & 0.660 \\
\hline $\begin{array}{l}\text { STOXX } \\
\text { Europe }\end{array}$ & 0.616 & 0.397 & 0.605 & 0.262 & 0.554 & 0.624 & 0.500 & 0.704 \\
\hline
\end{tabular}

capture heterogeneous investment preferences by decomposing the original time series into various frequency bands, establishing short-, medium-, and long-term horizons. The rationale behind the choice of wavelet decomposition via entropy analysis is to account for heterogeneous investment preferences of investors, which are not apparent in the scale-dependent information set. Market participants have heterogeneous investment and risk preferences and, therefore, distinctive term objectives and investment horizons. The wavelet transforms analysis decomposes time series into a set of discrete signals providing evidence related to the frequency domain of the series. To examine the temporal connectedness among underlying assets, we utilize a DCC-Student-t copula. The combination of wavelet transform analyses and time-varying DCC-Student-t copulas provide information that enhances our understanding of dependence among biotech assets and other asset classes in calm and volatile periods at different frequency horizons. Such information is important for identifying and implementing both risk assessment and portfolio management decisions across various investment horizons.

Our analyses indicate that biotech assets should be combined with commodities and forex markets to realize diversification benefits. We also conduct an extended analysis from an investor's perspective by examining the value at risk (VaR). We find that over the pre-2000 period, VaRs are higher for biotech assets, whereas over the post-2000 period VaRs have declined significantly with few periods of abrupt changes, especially during the Iraq war and the global financial crisis in line with Thakor et al. (2017). Furthermore, we find that VaRs 
decline significantly with an increase in the investment horizon, which suggests the meanreverting behavior of assets. Our findings have implications for investors and other capital market participants to design trading strategies. In summary, our analyses demonstrate that biotech assets have delivered solid financial returns (see Table 1) and offer diversification benefits (see Table 7) from 1995 to 2019. These results contradict more pessimistic findings by Fagnan et al. (2013), Fernandez et al. (2012), and Gopalakrishnan et al. (2008).

Acknowledgements Authors are thankful to the Trinity Business School, University of Dublin for the academic facilities provided to Gazi S. Uddin during his stay at Trinity where important parts of this research work was completed.Gazi Salah Uddin is thankful for the Visiting Fellow Programme Grant provided by the University of Jyväskylä, Finland.

Funding Open Access funding provided by Inland Norway University Of Applied Sciences.

Data availability The data that support the findings of this study are available from Datastream (published by Thomson Reuters Eikon), but restrictions apply to the availability of these data, which were used under license for the current study, and so are not publicly available. Data are, however, available from the authors upon reasonable request and with the permission of Thomson Reuters. The MATLAB code that replicates our findings is available from the authors.

Open Access This article is licensed under a Creative Commons Attribution 4.0 International License, which permits use, sharing, adaptation, distribution and reproduction in any medium or format, as long as you give appropriate credit to the original author(s) and the source, provide a link to the Creative Commons licence, and indicate if changes were made. The images or other third party material in this article are included in the article's Creative Commons licence, unless indicated otherwise in a credit line to the material. If material is not included in the article's Creative Commons licence and your intended use is not permitted by statutory regulation or exceeds the permitted use, you will need to obtain permission directly from the copyright holder. To view a copy of this licence, visit http://creativecommons.org/licenses/by/4.0/.

\section{References}

Adrian, T., \& Brunnermeier, M. K. (2016). CoVaR. American Economic Review, 106(7), 1705-1741.

Aguiar-Conraria, L., \& Soares, M. J. (2014). The continuous wavelet transform: Moving beyond uni-and bivariate analysis. Journal of Economic Surveys, 28(2), 344-375.

Arouri, M. E. H., Jouini, J., \& Nguyen, D. K. (2011). Volatility spillovers between oil prices and stock sector returns: Implications for portfolio management. Journal of International Money and Finance, 30(7), 1387-1405.

Baur, D. G., \& Lucey, B. M. (2010). Is gold a hedge or a safe haven? An analysis of stocks, bonds and gold. Financial Review, 45(2), 217-229.

Boyer, M. M., \& Filion, D. (2007). Common and fundamental factors in stock returns of Canadian oil and gas companies. Energy Economics, 29(3), 428-453.

Carvalho, C. M., Lopes, H. F., \& McCulloch, R. E. (2018). On the long-run volatility of stocks. Journal of the American Statistical Association, 113(523), 1050-1069. https://doi.org/10.1080/01621459.2017. 1407769

Coifman, R. R., \& Donoho, D. L. (1995). Translation-invariant de-noising. Wavelets and statistics (pp. 125-150). Springer.

Daubechies, I. (1992). Ten lectures on wavelets. SIAM. https://doi.org/10.2307/3620105

Engle, R. (2002). Dynamic conditional correlation: A simple class of multivariate generalized autoregressive conditional heteroskedasticity models. Journal of Business and Economic Statistics, 20(3), 339-350. https://doi.org/10.1198/073500102288618487

Fabozzi, F. J., \& Francis, J. C. (1978). Beta as a random coefficient. Journal of Financial and Quantitative Analysis, 13(1), 101-116.

Fagnan, D. E., Fernandez, J. M., Lo, A. W., \& Stein, R. M. (2013). Can financial engineering cure cancer? American Economic Review, 103, 406-411. https://doi.org/10.1257/aer.103.3.406

Fama, E. F., \& French, K. R. (1993). Common risk factors in the returns on stocks and bonds. Journal of Financial Economics, 33(1), 3-56. 
Fernandez, J. M., Stein, R. M., \& Lo, A. W. (2012). Commercializing biomedical research through securitization techniques. Nature Biotechnology. https://doi.org/10.1038/nbt.2374

Gençay, R., Selçuk, F., \& Whitcher, B. (2001). An introduction to wavelets and other filtering methods in finance and economics. Academic press.

Glosten, L. R., Jagannathan, R., \& Runkle, D. E. (1993). On the relation between the expected value and the volatility of the nominal excess return on stocks. The Journal of Finance. https://doi.org/10.2307/ 2329067

Gopalakrishnan, S., Scillitoe, J. L., \& Santoro, M. D. (2008). Tapping deep pockets: The role of resources and social capital on financial capital acquisition by biotechnology firms in biotech-pharma alliances. Journal of Management Studies, 45(8), 1354-1376. https://doi.org/10.1111/j.1467-6486.2008.00777.x

Jana, R. K., Ghosh, I., \& Das, D. (2021). A differential evolution-based regression framework for forecasting bitcoin price. Annals of Operations Research. https://doi.org/10.1007/s10479-021-04000-8

Joe, H. (1997). Multivariate models and multivariate dependence concepts. CRC Press.

Kahraman, E., \& Unal, G. (2016). Multiple wavelet coherency analysis and forecasting of metal prices. arXiv preprint arXiv: https://arxiv.org/abs/1602.01960.

Kılıç, D. K., \& Uğur, Ö. (2018). Multiresolution analysis of S\&P500 time series. Annals of Operations Research, 260(1), 197-216.

Kroner, K. F., \& Ng, V. K. (1998). Modeling asymmetric movements of asset prices. Review of Financial Studies, 11(04), 817-844.

Kroner, K. F., \& Sultan, J. (1993). Time-varying distributions and dynamic hedging with foreign currency futures. The Journal of Financial and Quantitative Analysis, 28(4), 535. https://doi.org/10.2307/2331164

Lintner, J. (1965). The valuation of risk assets and the selection of risky investments in stock portfolios and capital budgets. Review of Economics and Statistics, 47, 13-37.

Markowitz, H. (1952). Portfolio selection. Journal of Finance, 7(1), 77-91.

Mergner, S., \& Bulla, J. (2008). Time-varying beta risk of pan-European industry portfolios: A comparison of alternative modeling techniques. The European Journal of Finance, 14(8), 771-802.

Mestre, R. (2021). A wavelet approach of investing behaviors and their effects on risk exposures. Financial Innovation, 7(1), 1-37.

Mossin, J. (1966). Equilibrium in a capital asset market. Econometrica, 34(4), 768-783.

Nason, G. P., \& Sachs, R. V. (1999). Wavelets in time-series analysis. Philosophical Transactions of the Royal Society of London. Series A: Mathematical, Physical and Engineering Sciences, 357(1760), 2511-2526.

Nason, G. P., \& Silverman, B. W. (1995). The stationary wavelet transform and some statistical applications (pp. 281-299). New York: Springer.

Patton, A. J. (2006). Modelling asymmetric exchange rate dependence. International Economic Review, 47(2), 527-556. https://doi.org/10.1111/j.1468-2354.2006.00387.x

Percival, D. B., \& Walden, A. T. (2000). Wavelet methods for time seriesanalysis. Cambridge University Press. https://doi.org/10.1017/cbo9780511841040

Pesquet, J. C., Krim, H., Carfantan, H., \& Group, S. S. (1994). Time Invariant Orthonormal Wavelet Representations. IEEE Trans. Signal Processing. https://ieeexplore.ieee.org/abstract/document/533717/. Accessed March 312020

Shahzad, S. J. H., Bouri, E., Rehman, M. U., Naeem, M. A., \& Saeed, T. (2021). Oil price risk exposure of bric stock markets and hedging effectiveness. Annals of Operations Research. https://doi.org/10.1007/ s10479-021-04078-0

Sharpe, W. F. (1964). Capital asset prices: A theory of market equilibrium under conditions of risk. Journal of Finance, 19(3), 425-442.

Sklar, A. (1959). Fonctions de reparition a n dimensions et leurs marges. Publications De L'institute De Statistique De L'universite De Paris, 8, 229-231.

Thakor, R. T., Anaya, N., Zhang, Y., Vilanilam, C., Siah, K. W., Wong, C. H., \& Lo, A. W. (2017). Just how good an investment is the biopharmaceutical sector? Nature Biotechnology. https://doi.org/10.1038/nbt. 4023

Tzagkarakis, G., \& Maurer, F. (2020). An energy-based measure for long-run horizon risk quantification. Annals of Operations Research, 289(2), 363-390.

Yan, Z., Chen, Z., Consigli, G., Liu, J., \& Jin, M. (2020). A copula-based scenario tree generation algorithm for multiperiod portfolio selection problems. Annals of Operations Research, 292, 849-881.

Zhao, Y., Chang, S., \& Liu, C. (2015). Multifractal theory with its applications in data management. Annals of Operations Research, 234(1), 133-150.

Zhu, L., Wang, Y., \& Fan, Q. (2014). MODWT-ARMA model for time series prediction. Applied Mathematical Modelling, 38(5-6), 1859-1865.

Zou, Y., Yu, L., \& He, K. (2015). Wavelet entropy based analysis and forecasting of crude oil price dynamics. Entropy, 17(10), 7167-7184. 
Publisher's Note Springer Nature remains neutral with regard to jurisdictional claims in published maps and institutional affiliations. 